\title{
Segmentation and Classification of Hyperspectral Images Using Minimum Spanning Forest Grown From Automatically Selected Markers
}

\author{
Yuliya Tarabalka, Student Member, IEEE, Jocelyn Chanussot, Senior Member, IEEE, and \\ Jón Atli Benediktsson, Fellow, IEEE
}

\begin{abstract}
A new method for segmentation and classification of hyperspectral images is proposed. The method is based on the construction of a minimum spanning forest (MSF) from region markers. Markers are defined automatically from classification results. For this purpose, pixelwise classification is performed, and the most reliable classified pixels are chosen as markers. Each classification-derived marker is associated with a class label. Each tree in the MSF grown from a marker forms a region in the segmentation map. By assigning a class of each marker to all the pixels within the region grown from this marker, a spectral-spatial classification map is obtained. Furthermore, the classification map is refined using the results of a pixelwise classification and a majority voting within the spatially connected regions. Experimental results are presented for three hyperspectral airborne images. The use of different dissimilarity measures for the construction of the MSF is investigated. The proposed scheme improves classification accuracies, when compared to previously proposed classification techniques, and provides accurate segmentation and classification maps.
\end{abstract}

Index Terms-Classification, hyperspectral images, marker selection, minimum spanning forest (MSF), segmentation.

\section{INTRODUCTION}

$\mathbf{I}$ MAGE CLASSIFICATION, which can be defined as identification of objects in a scene captured by a vision system, is one of the important tasks of a robotic system. On the one side, the procedure of accurate object identification is known to be more difficult for computers than for people [1]. On the other side, recently developed image acquisition systems (for instance, radar, lidar, and hyperspectral imaging technologies) capture more data from the image scene than a human vision

Manuscript received May 29, 2009; revised September 4, 2009; accepted November 3, 2009. Date of publication December 31, 2009; date of current version September 15, 2010. This work was supported in part by the Marie Curie Research Training Network "Hyper-I-Net." This paper was recommended by Associate Editor D. Goldgof.

Y. Tarabalka is with the Grenoble Images Speech Signals and Automatics Laboratory (GIPSA Lab), Grenoble Institute of Technology (INPG), 38402 Grenoble, France, and the Faculty of Electrical and Computer Engineering, University of Iceland, 107 Reykjavik, Iceland (e-mail: yuliya.tarabalka@hyperinet.eu).

J. Chanussot is with the Grenoble Images Speech Signals and Automatics Laboratory (GIPSA Lab), Grenoble Institute of Technology (INPG), 38402 Grenoble, France (e-mail: jocelyn.chanussot@ gipsa-lab.grenoble-inp.fr).

J. A. Benediktsson is with the Faculty of Electrical and Computer Engineering, University of Iceland, 107 Reykjavik, Iceland (e-mail: benedikt@hi.is).

Color versions of one or more of the figures in this paper are available online at http://ieeexplore.ieee.org.

Digital Object Identifier 10.1109/TSMCB.2009.2037132

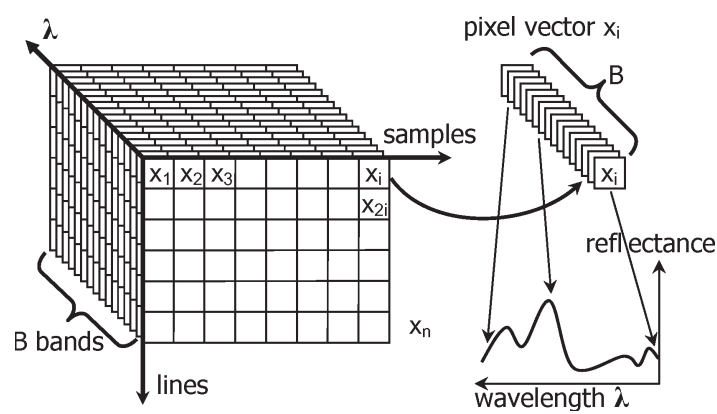

Fig. 1. Structure of a hyperspectral image.

system. Therefore, efficient processing systems must be developed in order to use these data for accurate image classification.

Hyperspectral imagery records a detailed spectrum of light arriving at each pixel [2]. Hyperspectral sensors measure the energy of the received light in tens or hundreds of narrow spectral bands (data channels) in each spatial position of the image (Fig. 1 shows the structure of a hyperspectral image). This rich information per pixel increases the capability to distinguish materials and objects and thus opens new perspectives for image classification. However, a large number of spectral channels, usually coupled with limited availability of reference data, ${ }^{1}$ present challenges to image analysis. While pixelwise classification techniques process each pixel independently without considering the information about spatial structures [3]-[5], further improvement of classification results can be achieved by considering spatial dependences between pixels, i.e., by performing spectral-spatial classification [6]-[11].

Segmentation is an exhaustive partitioning of the input image into homogeneous regions [12]. Segmentation techniques are a powerful tool to define spatial dependences. In previous works, we have performed unsupervised segmentation of hyperspectral images in order to define spatial structures [9], [13], [14]. Watershed, partitional clustering, and hierarchical segmentation techniques have been used for this purpose. Segmentation and pixelwise classification were performed independently, and then, the results were combined using a majority voting rule. Thus, every region from a segmentation map was considered as an adaptive homogeneous neighborhood for all the pixels within this region. The described technique led to a signification improvement of classification accuracies and provided more

\footnotetext{
${ }^{1}$ By reference data, we mean manually labeled pixels which are used for training classifiers followed by assessment of classification accuracies.
} 
homogeneous (less noisy) classification maps when compared to classification techniques using local neighborhoods in order to include spatial information into a classifier.

However, unsupervised image segmentation is a challenging task. Segmentation aims at dividing an image into homogeneous regions, but the measure of homogeneity is image dependent [12]. Depending on this measure, the process can result in undersegmentation (several regions are detected as one) or oversegmentation (one region is detected as several ones) of the image. In previous works [13], [14], we preferred oversegmentation to undersegmentation in order not to miss objects in the classification map. In this work, we aim to reduce oversegmentation and thus further improve segmentation and classification results. This can be achieved by using markers or region seeds [12], [15]. In previous studies, a marker (an internal marker) was defined as a connected component belonging to the image and associated with an object of interest [12], [15]-[17]. In our study, we define a marker as a set of image pixels (not necessarily connected; it can be composed of several spatially disjoint subsets of adjacent pixels) which is associated with one object in the image scene.

The problem of automatic marker selection has previously been discussed in the literature, mostly for gray-scale and color images. Markers are often defined by searching flat zones (i.e., connected components of pixels of constant gray-level value), zones of homogeneous texture, or image extrema [15]. Gómez et al. [18] applied histogram analysis to obtain a set of representative pixel values, and the markers were generated with all the image pixels with representative gray values. Jalba et al. [16] used connected operators filtering on the gradient image in order to select markers for a gray-scale diatom image. Noyel et al. [17], [19] performed classification of the hyperspectral image (using different techniques, such as Clara [20] and linear discriminant analysis) and then filtered the classification maps class by class, using morphological operators, in order to select large spatial regions as markers. Furthermore, the authors proposed to use random balls (connected sets of pixels of randomly selected sizes) extracted from these large regions as markers. In the discussed studies [16], [17], [19], the objective was to segment specific structures (blood cells, diatoms, glue occlusions, and cancerous growth).

In our study, the objective is to mark (select a marker for) each significant spatial object in the image. Here, by significant, we mean an object of at least one-pixel size that belongs to one of the classes of interest. As remote sensing images contain small and complex structures, automatic selection of markers is an especially challenging task.

In this paper, a new scheme for marker-based segmentation and classification of hyperspectral images is proposed. In particular, we propose to perform a probabilistic pixelwise classification first in order to choose the most reliable classified pixels as markers of spatial regions [21]. Furthermore, image pixels are grouped into a minimum spanning forest (MSF) [22], where each tree is rooted on a classification-derived marker. The decision to connect the pixel, which is not yet in the forest, to one of the trees in the forest is based on its similarity to one of the adjacent pixels already belonging to the forest. By assigning a class of the marker to all the pixels within the region grown from the considered marker, a spectralspatial classification map is obtained. Furthermore, the classification map is refined using the results of a pixelwise classification and a majority voting within the spatially connected regions [14].

The construction of an MSF belongs to graph-based approaches for image segmentation [22]-[25]. They introduce the Gestalt principles of perceptual grouping to the field of computer vision. The image is associated with a graph, the vertices of which correspond to the image entities (pixels or regions) and the edges correspond to relations between these entities. A weight associated with each edge indicates the (dis)similarity between two entities (pixels or regions). Morris et al. [23] have proposed to perform a graph-based image segmentation into $R$ regions by constructing a shortest spanning tree on the image graph and then removing the $R-1$ edges with the highest weight. Furthermore, several graph-cut-based algorithms have been developed for image segmentation [24], [25]. However, these methods perform unsupervised segmentation by splitting at each iteration one region into two subregions. This approach is fundamentally different from the work described in this paper. Several recent publications describe the use of an MSF rooted on markers for image segmentation [22], [26], [27]. However, the authors of these works do not investigate the problem of automatic marker selection. Their segmentation is based on markers provided by the user.

The proposed procedure of defining markers for each spatial object from probabilistic classification results and of building a spectral-spatial classification map for hyperspectral images by constructing an MSF rooted on classificationderived markers is a major contribution of this paper. Please note that, while, in previous studies, markers were used as seeds for image segmentation, in this paper, we introduce a new concept of the automatic marker-based spectral-spatial classification.

1) Markers are derived from probabilistic pixelwise classification results.

2) Each marker can be composed of several spatially disjoint subsets of adjacent pixels, and each marker has a class label.

3) By performing a region growing from the classificationderived markers, a spectral-spatial classification map is obtained.

Although the classification scheme proposed in this paper has been designed for hyperspectral data, the method is general and can successfully be applied for other types of data as well. Experimental results are demonstrated on hyperspectral airborne images recorded by the Airborne Visible/Infrared Imaging Spectrometer (AVIRIS) over Northwestern Indiana's Indiana Pine site and over the region surrounding the volcano Hekla in Iceland, and the image acquired by the Reflective Optics System Imaging Spectrometer (ROSIS) over the University of Pavia in Italy.

The outline of this paper is as follows. In the next section, a classification scheme based on an MSF rooted on markers is presented. Experimental results are discussed in Section III. Finally, conclusions are drawn in Section IV. 


\section{Segmentation and Classification Scheme}

The flowchart of the proposed segmentation and classification method is shown in Fig. 2. On the input, a B-band hyperspectral image is given, which can be considered as a set of $n$ pixel vectors $\mathbf{X}=\left\{\mathbf{x}_{j} \in \mathbb{R}^{B}, j=1,2, \ldots, n\right\}$. Classification consists in assigning each pixel to one of the $K$ classes of interest. In the following, each step of the proposed procedure is described.

\section{A. Pixelwise Classification}

The first step consists in performing a probabilistic pixelwise classification of the hyperspectral image. We propose to use a support vector machine (SVM) classifier [28] for this purpose. Other classifiers could be used. However, SVMs are extremely well suited to classify hyperspectral data [5], [29], [30]. We refer the reader to [5] and [28] for details on SVMs. The outputs of this step are the following:

1) classification map, containing class labels for each pixel;

2) probability map, containing probability estimates for each pixel to belong to the assigned class.

Two techniques for computing probability estimates for multiclass classification by pairwise coupling are described in [31]. We propose to use one of these methods, which is implemented in the LIBSVM library [32]. The objective is to estimate, for each pixel $\mathbf{x}$, the probabilities to belong to each class of interest

$$
\mathbf{p}=\left\{p_{k}=p(y=k \mid \mathbf{x}), k=1, \ldots, K\right\} .
$$

For this purpose, first, pairwise class probabilities $r_{i j} \approx$ $p(y=i \mid y=i$ or $j, \mathbf{x})$ are estimated using an improved implementation [33] of [34]

$$
r_{i j} \approx \frac{1}{1+e^{A \hat{f}+B}}
$$

where $A$ and $B$ are estimated by minimizing the negative loglikelihood function using known training data and decision values $\hat{f}$. Furthermore, the probabilities in (1) are computed by solving the following optimization problem:

$$
\begin{aligned}
& \min _{\mathbf{p}} \sum_{i=1}^{K} \sum_{j: j \neq i}\left(r_{j i} p_{i}-r_{i j} p_{j}\right)^{2} \\
& \text { subject to } \sum_{i=1}^{K} p_{i}=1, \quad p_{i} \geq 0 \forall i .
\end{aligned}
$$

This problem has a unique solution and can be solved by a simple linear system, as described in [31]. Finally, a probability map is constructed by assigning to each pixel the maximum probability estimate $\max \left(p_{k}\right), k=1, \ldots, K$.

\section{B. Selection of the Most Reliable Classified Pixels}

The aim of this step is to choose the most reliable classified pixels in order to define suitable markers. We propose to use probability estimates obtained as a result of the pixelwise classification for this purpose in order to keep the most reliable classified pixels as markers. A simple way of marker selection consists in thresholding the probability map. In other words, if the probability of the considered pixel belonging to the assigned class $k$ is higher than a given threshold, this pixel is selected to join the markers. In the resulting map of markers, each marker pixel is associated with the class defined by the pixelwise classifier. The marker pixels form connected components in the map of markers so that each connected component represents one marker. The main advantage of this technique of marker selection is its simplicity. However, this method has the following disadvantage: Each marker leads to one region in the segmentation map. Therefore, we need as many markers as the desired number of regions. However, if classes $k_{i}$ and $k_{j}$ are spectrally similar, pixels belonging to one of those classes have a quasi-equal probability to belong to each of them. From here, these classified pixels are not reliable. Therefore, we risk to lose the regions corresponding to either class $k_{i}$ or $k_{j}$ in the final segmentation map. This leads to undersegmentation, which is highly undesired.

To mitigate this problem, we propose the following method of marker selection [see the flowchart in Fig. 3(a)].

1) Perform a connected-component labeling of the pixelwise classification map. For this purpose, a classical connected-component algorithm using the union-find data structure can be used [35].

2) Analyze each connected region as follows.

- If a region is large enough, it should contain a marker, which is determined as $P \%$ of the pixels within the connected component with the highest probability estimates.

- If a region is small, it should lead to a marker only if it is very reliable; a potential marker is formed by pixels with probability estimates higher than a defined threshold.

The proposed procedure is deducted from the following analysis: Based on the results of our previous studies [9], [13], [14], it is common that almost no undersegmentation is present in a pixelwise classification map. Therefore, each connected spatial region from the classification map is analyzed if it corresponds most probably to the spatial structure or if it is rather a classification noise [see the illustrative example in Fig. 3(b)]. If the size of the component is large enough to consider it as a relevant region, the most reliable pixels within this region are selected as its marker. If a component contains only a few pixels, it is investigated if these pixels were classified to a particular class with a high probability. If this is the case, the considered connected component represents a small spatial structure. Thus, a marker associated with this region should be defined. Otherwise, the component is the consequence of classification noise, and we tend to eliminate it. Therefore, no marker within this component is selected. When performing labeling of connected components for a pixelwise classification map, we propose to use an eight-neighborhood connectivity.

For the proposed marker selection procedure, the following parameters must be chosen.

1) A parameter $M$ defining if a region is considered as being large or small. We propose to use a number of pixels in 


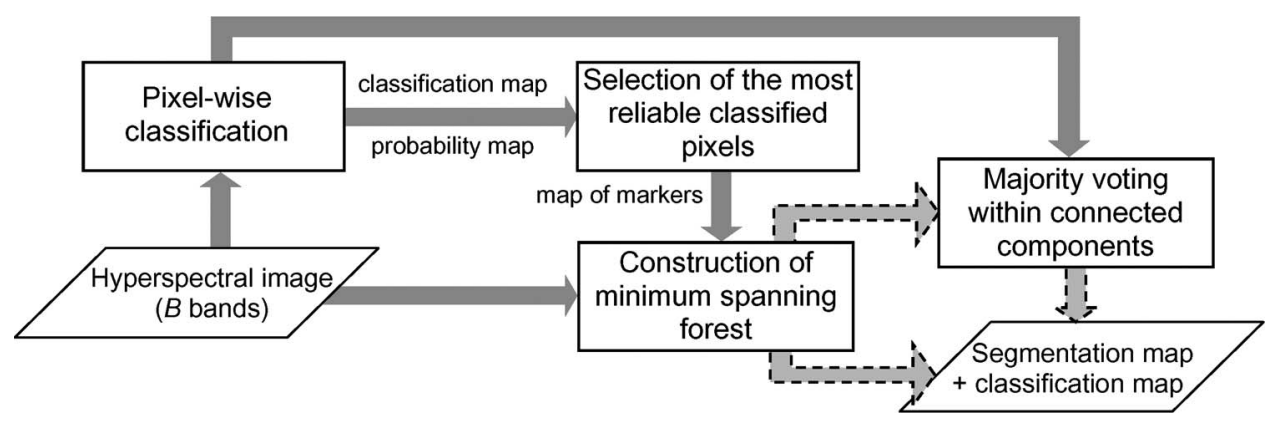

Fig. 2. Flowchart of the proposed segmentation and classification scheme.

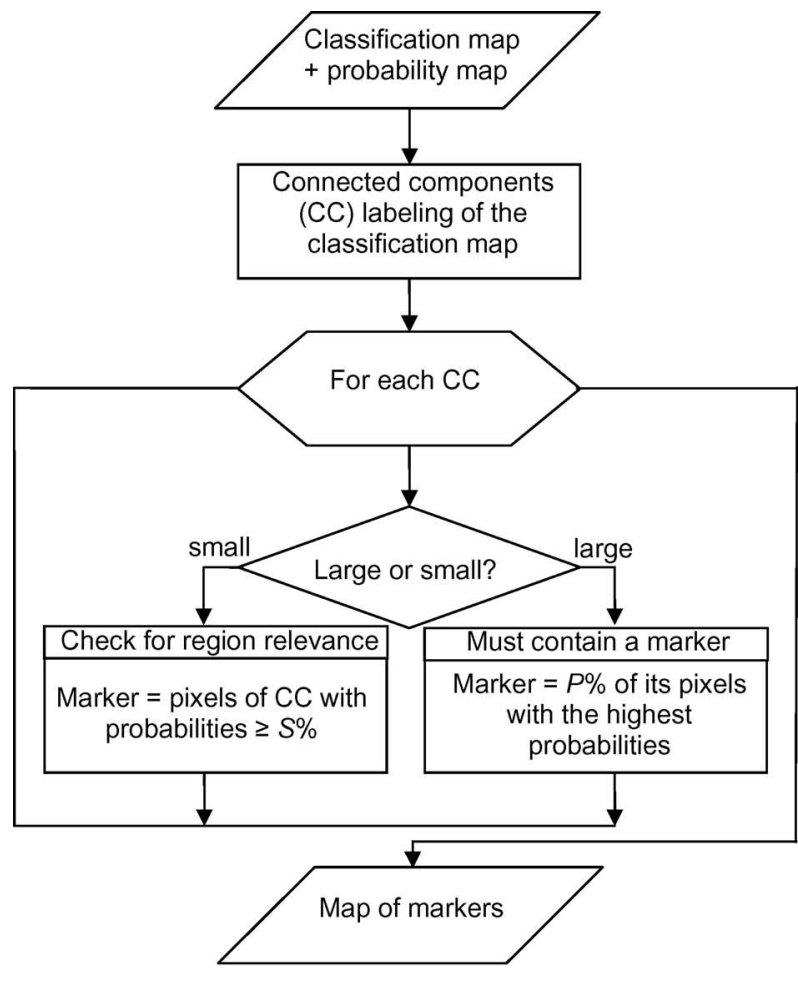

(a)

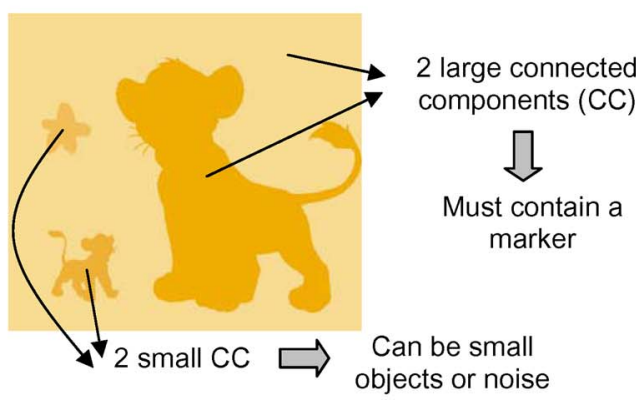

Result of the marker selection

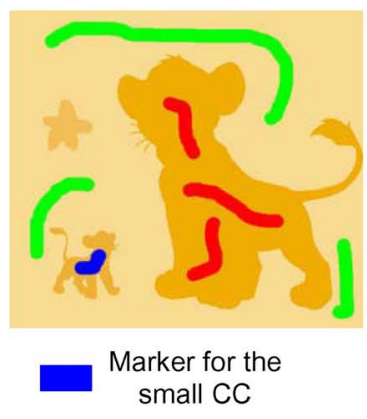

Marker for the first large CC

Marker for the second large CC

1. From $4 \mathrm{CC}$
3 markers are selected

2. Marker is not necessarily a connected set of pixels. It can be spatially split into several subsets

(b)

Fig. 3. (a) Flowchart of the proposed marker selection procedure. (b) Illustrative example of the marker selection.

the region (i.e., an area of the region) as a criterion of the region size. The threshold of the number of pixels defining if the region is large depends on the resolution of the image and typical sizes of the objects of interest. For instance, if the image of the volcano is considered (experimental results on the volcano image are illustrated in Section III), where the goal is to classify lavas of different eruption periods, it is known that the lava of different formations consists of large homogeneous regions. Therefore, it can be assumed that the regions representing structures (lavas) in the image scene have a size of at least $10 \mathrm{~km}^{2}$. Thus, for an airborne 20-m-resolution image, the threshold of $M=20$ pixels for dividing the regions in the groups of large/small ones can be chosen.

2) A parameter $P$, defining the percentage of pixels within the large region to be used as markers, depends on the previous parameter. Since a marker for the large region must be composed at least of one pixel, the following condition must be fulfilled: $P \geq 100 \% / M$.

3) The last parameter $S$, which is a threshold of probability estimates defining potential markers for a small region, depends on the probability of the presence of small structures in the image (which also depends on the image resolution and the classes of interest) and the importance of the potential small structures (i.e., what is the cost of losing the small structures in the classification map). For instance, if we are interested in determining regions of different lava formations in the volcano image, the small objects in the image may have no importance for us, and a high value of $S$ can be chosen. However, if the classification aims at determining regions of sick/damaged plants in the field, it may be important not to lose any small region of the damaged species. In this case, the threshold $S$ must be relaxed. 
In Section III-D, the dependence of the classification accuracies from the chosen parameters for the marker selection is investigated experimentally. As a conclusion, each connected set of pixels with the same class in the classification map provides either one or zero marker. One should stress that a marker is not necessarily a connected set of pixels: It can spatially be split into several subsets [see Fig. 3(b)].

\section{Construction of an MSF}

The previous two steps result in a map of markers defining regions of interest in the image. The next step consists in the grouping of all the image pixels into an MSF [22], where each tree is rooted on a classification-derived marker.

For this purpose, each pixel is considered as a vertex $v \in V$ of an undirected graph $G=(V, E, W)$, where $V$ and $E$ are the sets of vertices and edges, respectively, and $W$ is a mapping of the set of edges $E$ into $\mathbb{R}^{+}$. Each edge $e_{i, j} \in E$ of this graph connects a couple of vertices $i$ and $j$ corresponding to the neighboring pixels (in the following, we simply call vertices as pixels). Furthermore, a weight $w_{i, j}$ is assigned to each edge $e_{i, j}$, which indicates the degree of dissimilarity between two pixels connected by this edge. Different dissimilarity measures can be used for computing weights of edges, such as vector norms, Spectral Angle Mapper (SAM), and spectral information divergence (SID) [36].

The $L 1$ vector norm between two pixel vectors $\mathbf{x}_{i}=$ $\left(x_{i 1}, \ldots, x_{i B}\right)^{T}$ and $\mathbf{x}_{j}=\left(x_{j 1}, \ldots, x_{j B}\right)^{T}$ is given as

$$
L 1\left(\mathbf{x}_{i}, \mathbf{x}_{j}\right)=\sum_{b=1}^{B}\left|x_{i b}-x_{j b}\right| .
$$

The SAM distance between $\mathbf{x}_{i}$ and $\mathbf{x}_{j}$ determines the spectral similarity between two vectors by computing the angle between them. It is defined as

$$
\operatorname{SAM}\left(\mathbf{x}_{i}, \mathbf{x}_{j}\right)=\arccos \left(\frac{\sum_{b=1}^{B} x_{i b} x_{j b}}{\left[\sum_{b=1}^{B} x_{i b}^{2}\right]^{1 / 2}\left[\sum_{b=1}^{B} x_{j b}^{2}\right]^{1 / 2}}\right) .
$$

The SID measure [37] computes the discrepancy of probabilistic behaviors between the spectral signatures of two pixels. It is defined as

$$
\operatorname{SID}\left(\mathbf{x}_{i}, \mathbf{x}_{j}\right)=\sum_{b=1}^{B}\left\{q_{b}\left(\mathbf{x}_{i}\right) \log \left[\frac{q_{b}\left(\mathbf{x}_{i}\right)}{q_{b}\left(\mathbf{x}_{j}\right)}\right]+q_{b}\left(\mathbf{x}_{j}\right) \log \left[\frac{q_{b}\left(\mathbf{x}_{j}\right)}{q_{b}\left(\mathbf{x}_{i}\right)}\right]\right\}
$$

where

$$
q_{b}\left(\mathbf{x}_{i}\right)=\frac{x_{i b}}{\sum_{l=1}^{B} x_{i l}} .
$$

Furthermore, more complex dissimilarity measures for image segmentation have been proposed in [11] and [38].

Given a connected graph $G=(V, E)$, a spanning tree $T=$ $\left(V, E_{T}\right)$ of $G$ is a connected graph without cycles such that
$E_{T} \subset E$. A spanning forest $F=\left(V, E_{F}\right)$ of $G$ is a nonconnected graph without cycles such that $E_{F} \subset E$.

Given a graph $G=(V, E, W)$, the minimum spanning tree is defined as a spanning tree $T^{*}=\left(V, E_{T^{*}}\right)$ of $G$ such that the sum of the edge weights of $T^{*}$ is minimal

$$
T^{*} \in \arg \min _{T \in S T}\left\{\sum_{e_{i, j} \in E_{T}} w_{i, j}\right\}
$$

where $S T$ is a set of all spanning trees of $G$.

Given a graph $G=(V, E, W)$, the $M S F$ rooted on a set of $m$ distinct vertices $\left\{t_{1}, \ldots, t_{m}\right\}$ consists in finding a spanning forest $F^{*}=\left(V, E_{F^{*}}\right)$ of $G$, such that each distinct tree of $F^{*}$ is grown from one root $t_{i}$, and the sum of the edge weights of $F^{*}$ is minimal [22]

$$
F^{*} \in \arg \min _{F \in S F}\left\{\sum_{e_{i, j} \in E_{F}} w_{i, j}\right\}
$$

where $S F$ is a set of all spanning forests of $G$ rooted on $\left\{t_{1}, \ldots, t_{m}\right\}$.

In order to obtain the MSF rooted on markers, $m$ additional vertices $t_{i}, i=1, \ldots, m$, are introduced. Each extra vertex $t_{i}$ is connected by the edge with a null weight to the pixels representing a marker $i$. Furthermore, an additional root vertex $r$ is added and is connected by the null-weight edges to the vertices $t_{i}$. The minimum spanning tree of the constructed graph induces an MSF in $G$, where each tree is grown on a vertex $t_{i}$; the MSF is obtained after removing the vertex $r$. An example of the construction of the MSF rooted on markers is shown in Fig. 4. Prim's algorithm can be used for building the MSF (see Algorithm 1) [39]. The efficient implementation of the algorithm using a binary min heap (for the implementation of a min-priority queue) is possible [40]; the resulting time complexity of the algorithm is $O(|E| \log |V|)$.

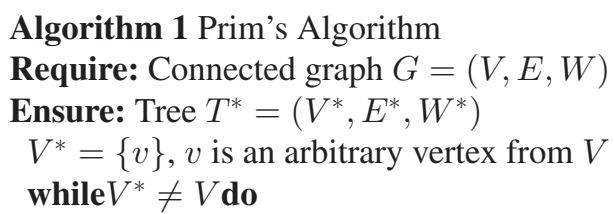

Choose edge $e_{i, j} \in E$ with minimal weight such that $i \in$ $V^{*}$ and $j \notin V^{*}$

$$
\begin{gathered}
V^{*}=V^{*} \cup\{j\} \\
E^{*}=E^{*} \cup\left\{e_{i, j}\right\} \\
\text { end while }
\end{gathered}
$$

Each tree in the MSF forms a region in the segmentation map (by mapping the resulting graph onto an image). Finally, a spectral-spatial classification map is obtained by assigning the class of each marker to all the pixels grown from this marker.

Thus, the proposed procedure of the construction of an MSF from region markers is a region growing method, which consists of the following steps: First, seed regions are chosen to belong to the segmentation and classification maps. Then, at each iteration, a new pixel $i$ is added to the segmentation and classification maps so that the dissimilarity criterion between 


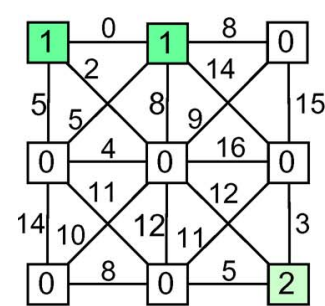

(a)

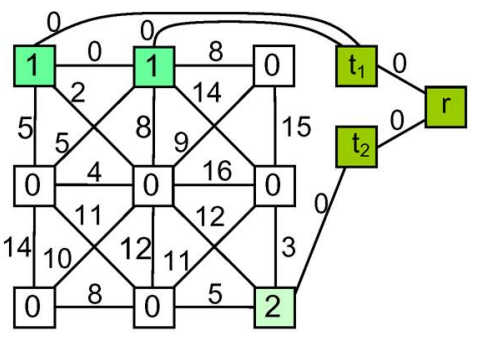

(b)

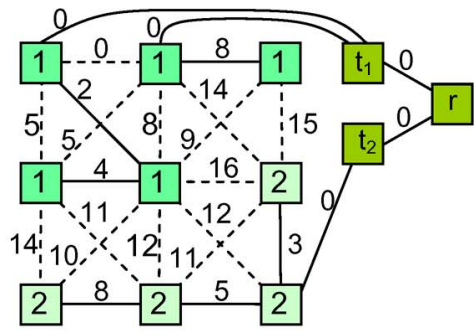

(c)

Fig. 4. Example of the construction of an MSF rooted on markers. (a) Original image graph $G$, where colored vertices represent markers 1 and 2; nonmarker pixels are denoted by " 0. ." (b) Addition of extra vertices $t_{1}, t_{2}$, and $r$ to the graph. (c) Minimum spanning tree of the graph presented in (b); after removing the vertex $r$, an MSF is obtained, where each tree grown from the vertex $t_{i}$ forms a region in the segmentation map.

this pixel and one of the pixels $j$ already belonging to the segmentation/classification map is minimal. When including the new pixel to the classification map, a class of the pixel $j$ is assigned to the pixel $i$.

\section{Majority Voting Within Connected Components}

Although the most reliable classified pixels are selected as markers, it may happen that a marker is classified to the wrong class. In this case, all the pixels within the region grown from this marker risk to be wrongly classified. In order to make the proposed classification scheme more robust, we propose to postprocess the classification map by applying a simple majority voting technique which has shown good performances for spectral-spatial classification [13], [14], [41]. For this purpose, connected-component labeling is applied on the obtained spectral-spatial classification map (using a four-neighborhood connectivity). Furthermore, for every connected component (region), all the pixels are assigned to the most frequent class when analyzing a pixelwise classification map within this region.

Note that an eight-neighborhood connectivity was used for the construction of an MSF, whereas a four-neighborhood connectivity was used for the majority voting. The use of the eightneighborhood connectivity in the first case allows obtaining a more accurate (refined) segmentation map, without rough borders. Since an MSF is built from the set of markers, the number of regions does not depend on the chosen connectivity. When performing the last majority voting step, the use of the four-neighborhood connectivity results in a larger or the same number of connected components as the use of the eightneighborhood connectivity. Therefore, the possible undersegmentation can be corrected in this step. One region from a segmentation map can be split into two connected regions when using the four-neighborhood connectivity. Furthermore, these two regions can be assigned to two different classes by the majority voting procedure.

\section{EXPERIMENTAL RESULTS}

Three different data sets were used for the experiments, with different contexts (agricultural, volcano, and urban areas) and acquired by different sensors (AVIRIS and ROSIS airborne imaging spectrometers). These data sets and the corresponding results are presented in the next three sections.

\section{A. Classification of the Indiana Image}

The Indiana image is of a vegetation area that was recorded by the AVIRIS sensor over the Indian Pine test site in Northwestern Indiana. The image has spatial dimensions of 145 by 145 pixels and a spatial resolution of $20 \mathrm{~m} /$ pixel. Twenty water absorption bands have been removed [42], and a 200-band image was used for the experiments. Sixteen classes of interest are considered, which are detailed in Table I, with a number of samples for each class in the reference data. A three-band false color image and the reference data are shown in Fig. 5. We have chosen randomly 50 samples for each class from the reference data as training samples, except for classes alfalfa, grass/pasture-mowed, and oats. These classes contain a small number of samples in the reference data. Therefore, only 15 samples for each of these classes were chosen randomly to be used as training samples. The remaining samples comprised the test set.

A pixelwise classification on the 200-band Indiana image was performed, using the multiclass one versus one SVM classifier with the Gaussian radial basis function (RBF) kernel. The optimal parameters $C$ and $\gamma$ were chosen by fivefold cross validation: $C=128$ and $\gamma=2^{-6}$. Global and class-specific accuracies are presented in Table I. Fig. 5 shows the obtained classification and probability maps.

Furthermore, a map of markers was created. For this purpose, labeling of connected components on the pixelwise classification map was performed, using the eight-neighborhood connectivity. For each connected component, the following are observed.

1) If it contained more than $M=20$ pixels, $P=5 \%$ of its pixels with the highest probability estimates were selected as a marker for this component.

2) Otherwise, if a connected component contained pixels with the corresponding probability estimates not lower than the threshold $S$, these pixels were used as a marker.

In order to define a threshold $S$, the probability estimates for the whole image were sorted, and $S$ was chosen equal to the lowest probability within the highest $T=2 \%$ of all probability estimates. The parameters for marker selection were chosen based on the following analysis: It is known that the image consists of the fields of different types of crops, i.e., large homogeneous regions. In the reference data, the class oats is represented by the smallest field of a size of 20 pixels. Therefore, the classification procedure must be able to 
TABLE I

Information Classes, Number of LABElEd SAMPles (No. OF SAMP.), AND ClassifiCATION ACCURACIES IN PERCENTAGE FOR THE Indiana Image: Overall ACCuracy (OA), Average ACCuracy (AA), Kappa CoefFicient $(\kappa)$, And Class-Specific ACCuracies; "MV" Means InCluding a Majority Voting Step

\begin{tabular}{|c|c|c|c|c|c|c|c|c|c|c|}
\hline & \multirow{2}{*}{$\begin{array}{l}\text { No.of } \\
\text { Samp. }\end{array}$} & \multirow{2}{*}{ SVM } & \multicolumn{6}{|c|}{ Proposed Method (Using L1, SAM, SID Dissimilarity Measures) } & \multirow{2}{*}{ M-WH } & \multirow{2}{*}{$\mathrm{WH}+\mathrm{MV}$} \\
\hline & & & L1 & $\mathrm{L} 1+\mathrm{MV}$ & SAM & $\mathrm{SAM}+\mathrm{MV}$ & SID & SID+MV & & \\
\hline$\overline{\mathrm{OA}}$ & - & 78.17 & 86.94 & 88.10 & 88.41 & 91.80 & 89.21 & 91.53 & 85.99 & 86.63 \\
\hline AA & - & 85.97 & 88.66 & 91.82 & 91.57 & 94.28 & 92.08 & 94.14 & 86.95 & 91.61 \\
\hline$\kappa$ & - & 75.33 & 85.03 & 86.38 & 86.71 & 90.64 & 87.63 & 90.33 & 83.98 & 84.83 \\
\hline Corn-no till & 1434 & 78.18 & 89.31 & 89.45 & 90.97 & 93.21 & 91.26 & 91.26 & 80.35 & 94.22 \\
\hline Corn-min till & 834 & 69.64 & 66.71 & 62.63 & 69.52 & 96.56 & 74.62 & 95.79 & 71.94 & 78.06 \\
\hline Corn & 234 & 91.85 & 64.13 & 99.46 & 95.65 & 95.65 & 95.65 & 95.65 & 73.37 & 88.59 \\
\hline Soybeans-no till & 968 & 82.03 & 99.35 & 93.04 & 98.04 & 93.91 & 98.26 & 94.12 & 98.91 & 96.30 \\
\hline Soybeans-min till & 2468 & 58.95 & 80.81 & 81.02 & 81.97 & 81.97 & 82.18 & 82.18 & 80.48 & 68.82 \\
\hline Soybeans-clean till & 614 & 87.94 & 86.17 & 97.52 & 85.99 & 97.16 & 86.35 & 97.52 & 84.75 & 90.78 \\
\hline Alfalfa & 54 & 74.36 & 92.31 & 92.31 & 94.87 & 94.87 & 94.87 & 94.87 & 94.87 & 94.87 \\
\hline Grass/pasture & 497 & 92.17 & 93.51 & 93.51 & 94.63 & 94.63 & 94.63 & 94.63 & 95.30 & 95.08 \\
\hline Grass/trees & 747 & 91.68 & 91.82 & 97.27 & 92.40 & 97.27 & 92.40 & 97.27 & 92.97 & 97.99 \\
\hline Grass/pasture-mowed & 26 & 100 & 100 & 100 & 100 & 100 & 100 & 100 & 100 & 100 \\
\hline Hay-windrowed & 489 & 97.72 & 99.77 & 99.77 & 99.77 & 99.77 & 99.77 & 99.77 & 99.54 & 99.54 \\
\hline Oats & 20 & 100 & 100 & 100 & 100 & 100 & 100 & 100 & 100 & 100 \\
\hline Wheat & 212 & 98.77 & 99.38 & 99.38 & 99.38 & 99.38 & 99.38 & 99.38 & 99.38 & 99.38 \\
\hline Woods & 1294 & 93.01 & 98.47 & 98.47 & 97.59 & 99.68 & 99.52 & 99.52 & 99.36 & 97.11 \\
\hline Bldg-Grass-Tree-Drives & 380 & 61.52 & 61.21 & 69.70 & 68.79 & 68.79 & 68.79 & 68.79 & 55.45 & 69.39 \\
\hline Stone-steel towers & 95 & 97.78 & 95.56 & 95.56 & 95.56 & 95.56 & 95.56 & 95.56 & 64.44 & 95.56 \\
\hline
\end{tabular}

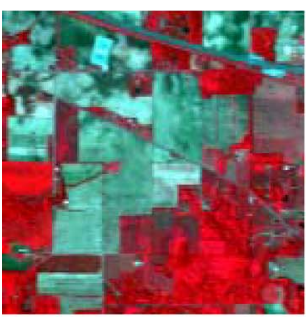

(a)

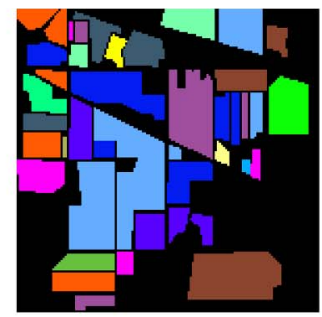

(b)

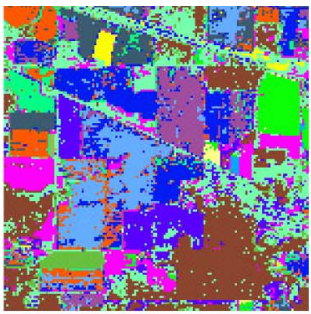

(c)

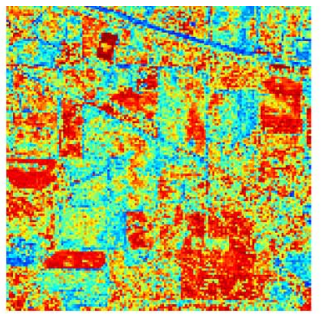

(d)

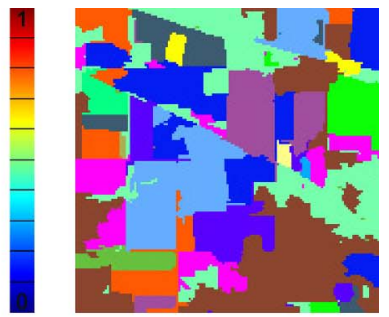

(e)

(f)

Fig. 5. Indiana image. (a) Three-band color composite (837, 636, and 537 nm). (b) Reference data:Corn-no till, Corn-min till, Corn, Soybeans-no till, Soybeans-min till, Soybeans-clean till, Alfalfa, Grass/pasture, Grass/trees, Grass/pasture-mowed, Hay-windrowed, Oats, Wheat, Woods, Bldg-Grass-Tree-Drives, Stone-steel towers. (c) Pixelwise classification map. (d) Probability map (probability estimates for each pixel to belong to the assigned class). (e) Scale of colors to represent the probability estimates in a probability map, from $0 \%$ probability at the bottom to $100 \%$ probability at the top. (f) Classification map obtained by the proposed scheme, using the SAM dissimilarity measure and including a majority voting step.

recognize the 20-pixel regions. We select $M=20$. The parameter $P$ is computed as $P=100 \% / M=5 \%$. This means that, for a region of 20 pixels, a one-pixel marker is selected. The last parameter $T$ is chosen to be low since we know that the image does not contain small spatial structures. The correctness and robustness of this theoretical deduction of parameters are proved in Section III-D.

From 2250 connected components in the classification map, 107 markers were selected. Of the marker pixels presented in the reference data, $95 \%$ are correctly classified. In the next step, the image pixels were grouped into the MSF, built from the selected markers. We have investigated the use of different dissimilarity measures: the $L 1$ vector norm, ${ }^{2}$ the SAM, and the SID measures [36]. When the class of each marker was assigned to all the pixels of the corresponding tree, the spectralspatial classification maps were obtained. Finally, the obtained classification maps were combined with the pixelwise classifi-

\footnotetext{
${ }^{2}$ We have also considered the $L 2$ vector norm as a dissimilarity measure for the construction of the MSF. The corresponding classification accuracies are not given in this paper because of space limitations. These accuracies are mostly nonsignificantly lower than the ones obtained when using the $L 1$ norm.
}

cation map, using the majority voting technique, as described in Section II-D.

Table I summarizes the accuracies of the pixelwise SVM and the proposed classification method (before and after the majority voting step). In order to compare performances of the proposed technique with the previously proposed methods, we have included results of a classification using majority vote within the adaptive neighborhoods defined by watershed segmentation $(W H+M V)$ [13], as well as classification results obtained by performing watershed segmentation ${ }^{3}$ from the same set of markers $(M-W H)$ [21]; these are recently proposed advanced techniques for spectral-spatial classification of hyperspectral images.

First of all, almost no oversegmentation is present in the obtained segmentation map (since one marker led to one region, a segmentation map contains 107 regions). As can be seen from Table I, both the global and most of the class-specific

${ }^{3}$ In [21], watershed segmentation is computed using the classical paradigm of the morphological image segmentation [19]: A gradient of the image is computed; then, a minima imposition technique is applied, followed by the watershed algorithm based on flooding simulations. 
accuracies are improved by the proposed method. The majority voting step additionally improves most of the accuracies (except for the class soybeans-no till). The best global accuracies are achieved by the proposed method when using the SAM dissimilarity measure and including a majority voting step [the corresponding classification map is shown in Fig. 5(f)]. In this case, the overall accuracy is improved by 13.6 percentage points and the average accuracy by 8.3 percentage points when compared to the pixelwise classification. However, the use of other dissimilarity measures also leads to high accuracies; the highest accuracies for 7 of the 16 classes are achieved when using each of the proposed measures.

Furthermore, McNemar's test was performed in order to evaluate the statistical significance of differences in classification accuracies between the most accurate classification map $(S A M+M V)$ and other classification maps [43]. According to the results of the test, the $S A M+M V$ classification accuracies are significantly different (here and in the following, we use $1 \%$ level of significance when reporting results of McNemar's test) from the accuracies of any other classification method applied for the Indiana image. The improvement of the accuracies after the majority voting step is also significant when using any of the applied dissimilarity measures.

\section{B. Classification of the Hekla Image}

The Hekla image was acquired by the AVIRIS sensor over the region surrounding the central volcano Hekla in Iceland [44]. The AVIRIS sensor operates in the wavelength range from 0.4 to $2.4 \mu \mathrm{m}$ and utilizes four spectrometers collecting 224 data channels. During the data collection, spectrometer 4 was not working properly. The 64 data channels recorded by this spectrometer were deleted from the data, along with the first channels for the other three spectrometers (those channels were blank). Therefore, the 157 remaining data channels were used for the experiments. The considered image has spatial dimensions of 560 by 600 pixels. Twelve land cover classes of interest are considered, which are detailed in Table II, with a number of labeled samples for each class. Fig. 6 shows a three-band false color image and the reference data. Fifty samples for each class were randomly chosen from the reference data as training samples, and the rest of the samples were used as the test set.

A multiclass one versus one SVM classification on the original image was performed using the Gaussian RBF kernel. The parameters $C=100$ and $\gamma=0.1$ were determined by fivefold cross validation. Table II gives the classification accuracies, and the classification map is shown in Fig. 6(c).

In the next step, a map of markers was created, with the same parameters as for the Indiana image. Furthermore, segmentation and classification of the image were performed by constructing an MSF based on the selected markers. The obtained classification maps were further combined with the pixelwise classification map using the majority voting technique.

Table II gives the accuracies of the proposed classification method. As can be seen, both the global and most of the class-specific accuracies are improved when compared to the pixelwise classification. The majority voting step additionally improves the accuracies. As for the Indiana image, the best global accuracies are achieved when performing the proposed classification with the SAM dissimilarity measure and the majority voting step. Fig. 6(d) shows the corresponding classification map, which is much less noisy than a pixelwise classification map. In this case, the overall accuracy is improved by 10.4 percentage points and the average accuracy by 9.0 percentage points when compared to the pixelwise classification. According to the results of McNemar's test, all the obtained classification maps are significantly different.

\section{Classification of the University of Pavia Image}

The proposed scheme was also tested on the University of Pavia image of an urban area, acquired by the ROSIS-03 optical sensor. The image is 610 by 340 pixels, with a spatial resolution of $1.3 \mathrm{~m} /$ pixel and 103 spectral channels. The reference data contain nine classes of interest. More information about the image, with the number of test and training samples for each class, can be found in [14].

Segmentation and classification of the University of Pavia image were performed using the proposed scheme. The parameters for an SVM classification were chosen by fivefold cross validation: $C=128$ and $\gamma=0.125$. Marker selection was performed with the same parameters as for the two previous data sets. Table III summarizes the classification accuracies for a pixelwise and spectral-spatial classification. In order to compare the performances of the proposed method with the previously proposed techniques, we have included in the table the accuracies of the classification of the University of Pavia image using an SVM, principal components, and extended morphological profiles; results are taken from [45]. This method has been recently proposed by Benediktsson et al. [46] and is considered as one of the most advanced methods for spectralspatial classification of a multiband datum. Furthermore, the results of the spectral-spatial classification using majority voting within adaptive neighborhoods defined by spatial-based segmentation techniques are included. The following segmentation techniques are used for this purpose (leading to the best classification results among all the spatial-based methods): watershed segmentation $(W H+M V)$ [13] and recursive hierarchical segmentation (RHSEG), with the possibility of merging only adjacent regions $(R H S E G 0+M V)$ [9].

As can be seen from Table III, both the global and most of the class-specific accuracies are improved by the proposed method. The majority voting step additionally improves most of the accuracies. The best global accuracies are achieved by the proposed method when using the $L 1$ vector norm for measuring dissimilarity between pixels. The corresponding classification map is significantly more accurate than any other obtained classification map, according to the results of McNemar's test. In this case, the overall accuracy is improved by 10.1 percentage points and the average accuracy by 6.5 percentage points when compared to the pixelwise classification. Those accuracies are higher than the ones obtained by the previously proposed techniques given for comparison. The use of the other two measures also led to the high classification accuracies for most of the classes. For instance, the use of the SAM measure led to the best accuracy of classification for the class asphalt when 
TABLE II

Information Classes, Number of Labeled SAmples (No. of SAmp.), and Classification Accuracies in Percentage for the HeKla Image: Overall ACCURACy (OA), Average ACCURACy (AA), Kappa CoefFicient $(\kappa)$, ANd Class-Specific ACCURACIES; "MV" Means InCLUding a Majority Voting SteP

\begin{tabular}{l||c||c||c|c||c|c||c|c}
\hline \multicolumn{1}{l|}{} & \multirow{2}{*}{$\begin{array}{c}\text { No.of } \\
\text { Samp. }\end{array}$} & \multirow{2}{*}{ SVM } & \multicolumn{3}{c|}{ Proposed Method (Using L1, SAM, SID Dissimilarity Measures) } \\
\cline { 5 - 8 } & & L1 & L1+MV & SAM & SAM+MV & SID & SID+MV \\
\hline OA & - & 88.56 & 91.45 & 97.25 & 90.34 & $\mathbf{9 8 . 9 6}$ & 86.08 & 98.37 \\
AA & - & 89.44 & 94.11 & 96.36 & 94.89 & $\mathbf{9 8 . 4 5}$ & 91.44 & 98.25 \\
$\kappa$ & - & 86.91 & 90.25 & 96.81 & 89.04 & $\mathbf{9 8 . 8 0}$ & 84.31 & 98.11 \\
\hline Andesite lava 1970 & 342 & 88.36 & $\mathbf{1 0 0}$ & $\mathbf{1 0 0}$ & $\mathbf{1 0 0}$ & $\mathbf{1 0 0}$ & $\mathbf{1 0 0}$ & $\mathbf{1 0 0}$ \\
Andesite lava 1980 I & 708 & 87.25 & $\mathbf{1 0 0}$ & $\mathbf{1 0 0}$ & 92.11 & $\mathbf{1 0 0}$ & 75.57 & $\mathbf{1 0 0}$ \\
Andesite lava 1980 II & 1496 & 88.24 & 98.96 & 98.96 & 96.96 & 99.86 & 97.10 & $\mathbf{1 0 0}$ \\
Andesite lava 1991 I & 2739 & 84.94 & 79.17 & 98.77 & 73.19 & $\mathbf{9 9 . 5 5}$ & 65.79 & 97.21 \\
Andesite lava 1991 II & 410 & $\mathbf{9 3 . 3 3}$ & 86.67 & 86.67 & 88.89 & 88.89 & 86.94 & 86.94 \\
Andesite lava with moss cover & 1023 & 94.24 & $\mathbf{9 8 . 4 6}$ & $\mathbf{9 8 . 4 6}$ & $\mathbf{9 8 . 4 6}$ & $\mathbf{9 8 . 4 6}$ & $\mathbf{9 8 . 4 6}$ & $\mathbf{9 8 . 4 6}$ \\
Hyaloclastite formation & 684 & 87.54 & 82.02 & 81.86 & 99.53 & $\mathbf{9 9 . 6 8}$ & 82.65 & 99.53 \\
Lava covered with tephra and scoria & 700 & 91.69 & 98.31 & 98.31 & 95.08 & 97.38 & 97.08 & $\mathbf{9 9 . 2 3}$ \\
Rhyolite & 404 & 85.88 & 92.37 & 96.05 & 96.89 & $\mathbf{1 0 0}$ & 96.05 & $\mathbf{1 0 0}$ \\
Scoria & 550 & 74.20 & 93.40 & 97.20 & $\mathbf{9 7 . 6 0}$ & $\mathbf{9 7 . 6 0}$ & $\mathbf{9 7 . 6 0}$ & $\mathbf{9 7 . 6 0}$ \\
Firn and glacier ice & 458 & $\mathbf{1 0 0}$ & $\mathbf{1 0 0}$ & $\mathbf{1 0 0}$ & $\mathbf{1 0 0}$ & $\mathbf{1 0 0}$ & $\mathbf{1 0 0}$ & $\mathbf{1 0 0}$ \\
Snow & 713 & 97.59 & $\mathbf{1 0 0}$ & $\mathbf{1 0 0}$ & $\mathbf{1 0 0}$ & $\mathbf{1 0 0}$ & $\mathbf{1 0 0}$ & $\mathbf{1 0 0}$ \\
\hline
\end{tabular}

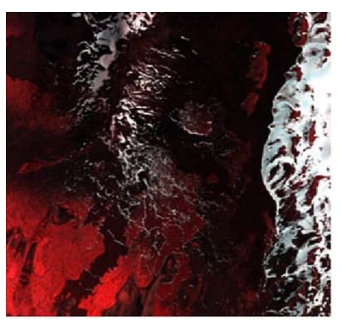

(a)

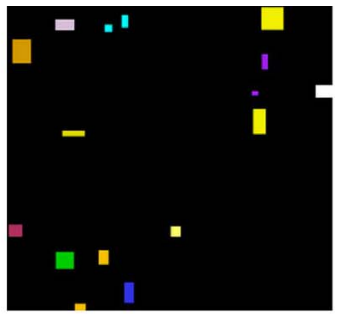

(b)

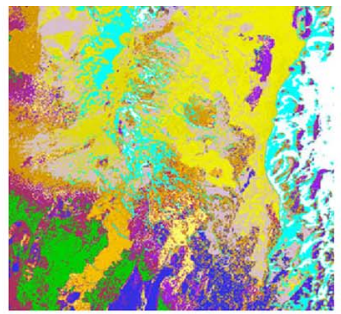

(c)

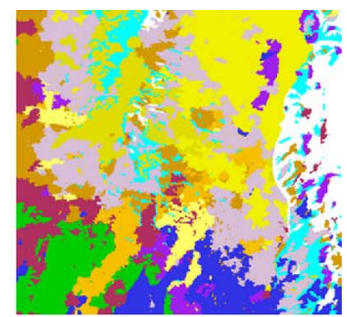

(d)

Fig. 6. Hekla image. (a) Three-band color composite (1125, 636, and $567 \mathrm{~nm})$. (b) Reference data: Andesite lava 1970, Andesite lava $1980 \mathrm{I}$, Andesite lava 1980 II, Andesite lava 1991 I, Andesite lava 1991 II, Andesite lava with moss cover, Hyaloclastite formation, Lava covered with tephra and scoria, Rhyolite, Scoria, Firn and glacier ice, and snow (white). (c) Pixelwise classification map. (d) Classification map obtained by the proposed scheme, using the SAM dissimilarity measure and including a majority voting step.

TABLE III

Classification Accuracies in Percentage for the University of Pavia Image: Overall Accuracy (OA), Average Accuracy (Aa), Kappa Coefficient $(\kappa)$, and Class-Specific Accuracies; "MV” Means Including a Majority Voting SteP

\begin{tabular}{|c|c|c|c|c|c|c|c|c|c|c|}
\hline & \multirow{2}{*}{ SVM } & \multicolumn{6}{|c|}{ Proposed Method (Using L1, SAM, SID Dissimilarity Measures) } & \multirow{2}{*}{$\begin{array}{l}\text { WH } \\
+\mathrm{MV}\end{array}$} & \multirow{2}{*}{$\begin{array}{c}\text { RHSEG0 } \\
+\mathrm{MV}\end{array}$} & \multirow{2}{*}{ EMP } \\
\hline & & L1 & $\mathrm{L} 1+\mathrm{MV}$ & SAM & $\mathrm{SAM}+\mathrm{MV}$ & SID & SID+MV & & & \\
\hline$\overline{\mathrm{OA}}$ & 81.01 & 84.14 & 91.08 & 78.62 & 83.94 & 77.97 & 83.38 & 85.42 & 90.00 & 85.22 \\
\hline AA & 88.25 & 92.35 & 94.76 & 85.90 & 88.01 & 85.94 & 89.27 & 91.31 & 94.15 & 90.76 \\
\hline$\kappa$ & 75.86 & 79.71 & 88.30 & 73.28 & 79.59 & 72.45 & 78.94 & 81.30 & 86.86 & 80.86 \\
\hline Asphalt & 84.93 & 93.05 & 93.16 & 95.78 & 96.88 & 95.69 & 96.13 & 93.64 & 73.33 & 95.36 \\
\hline Meadows & 70.79 & 72.30 & 85.65 & 62.61 & 70.92 & 61.60 & 68.63 & 75.09 & 88.73 & 80.33 \\
\hline Gravel & 67.16 & 89.15 & 89.15 & 94.66 & 77.69 & 94.27 & 95.21 & 66.12 & 97.47 & 87.61 \\
\hline Trees & 97.77 & 87.02 & 91.24 & 96.26 & 96.50 & 93.30 & 93.75 & 98.56 & 98.45 & 98.37 \\
\hline Metal sheets & 99.46 & 99.91 & 99.91 & 88.05 & 99.82 & 99.82 & 99.82 & 99.91 & 99.10 & 99.48 \\
\hline Bare soil & 92.83 & 97.11 & 99.91 & 85.13 & 99.80 & 83.53 & 99.76 & 97.35 & 98.43 & 63.72 \\
\hline Bitumen & 90.42 & 98.57 & 98.57 & 97.55 & 97.55 & 97.96 & 97.96 & 96.23 & 95.92 & 98.87 \\
\hline Bricks & 92.78 & 95.66 & 99.05 & 96.70 & 98.63 & 96.88 & 98.75 & 97.92 & 98.81 & 95.41 \\
\hline Shadows & 98.11 & 98.36 & 96.23 & 56.35 & 54.34 & 50.44 & 53.46 & 96.98 & 97.11 & 97.68 \\
\hline
\end{tabular}

compared to all the previous results found in the literature. However, the global accuracies are not as high as when using the $L 1$ vector norm mainly because of the low accuracy for the class shadows. The SAM distance is actually designed with the purpose that the poorly illuminated and more brightly illuminated pixels from the same class would be mapped to the same spectral angle despite the difference in illumination. It can be an explanation of the fact why the SAM distance led to the assimilation of the shadowed regions with the neighboring structures.

\section{Assessment of the Robustness of the Parameter Settings}

In Section II-B, the procedure of the choice of parameters for the marker selection procedure was explained. In this section, the dependence of the classification accuracies from the chosen parameters is investigated experimentally for the Indiana and the Hekla images.

First, the choice of the parameter $P$ is analyzed. For this purpose, the other two parameters were fixed as $M=20$ and $T=$ 2. Classification using the proposed scheme was performed, with the parameter $P$ varying from 5 to 100 . The $L 1$ vector 


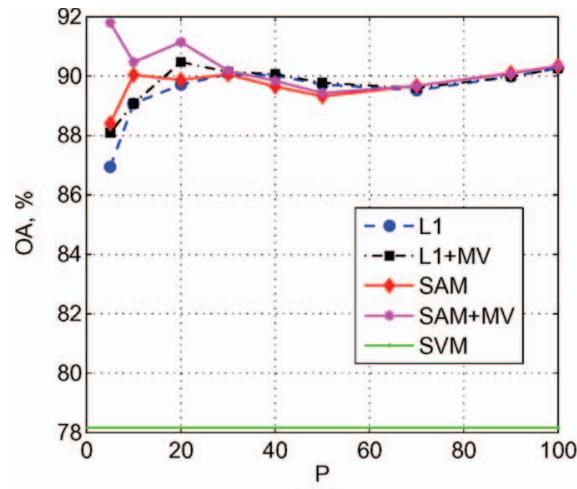

(a)

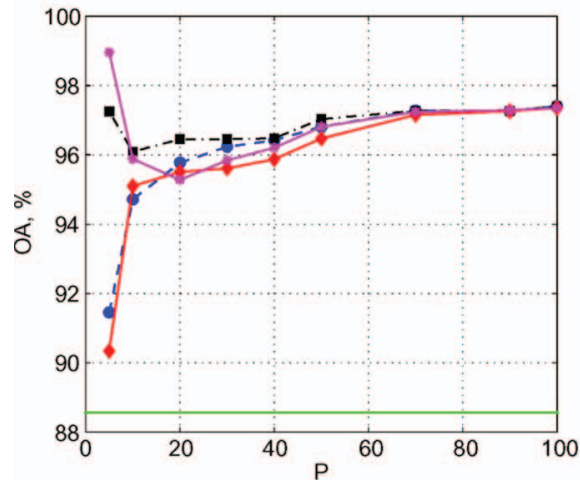

(d)

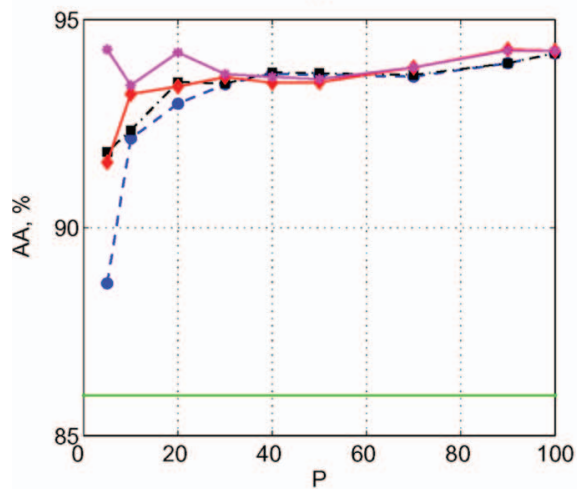

(g)

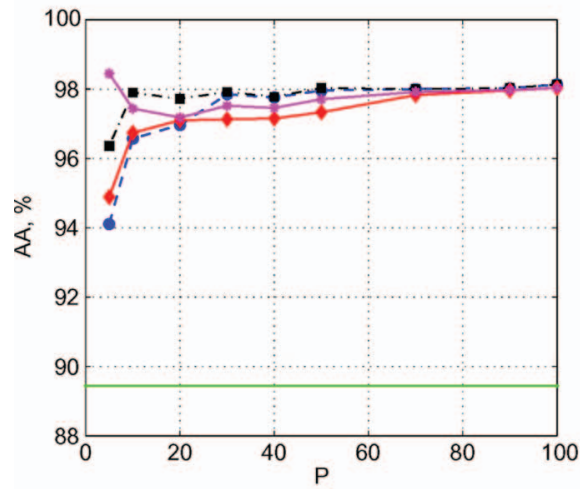

(i)

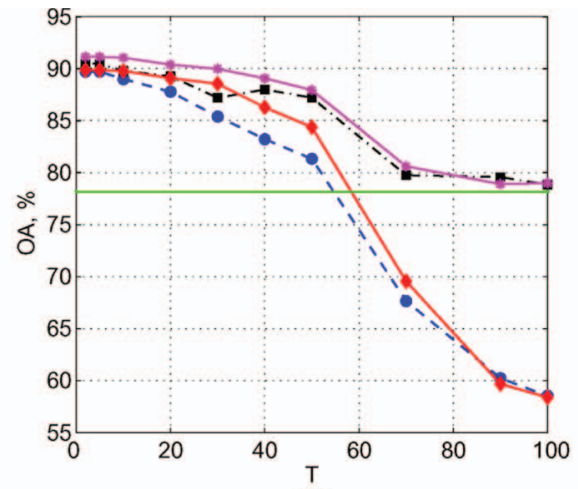

(b)

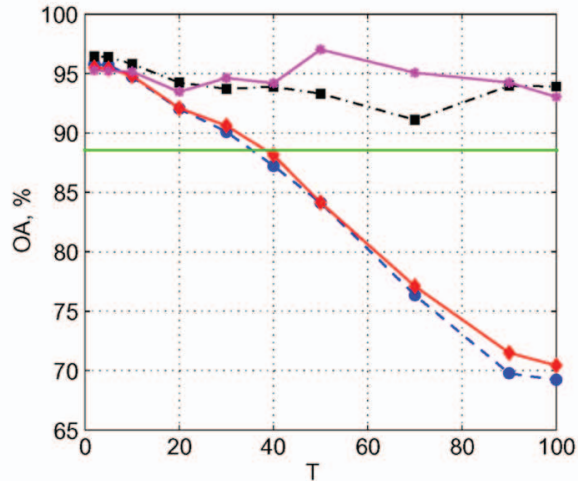

(e)

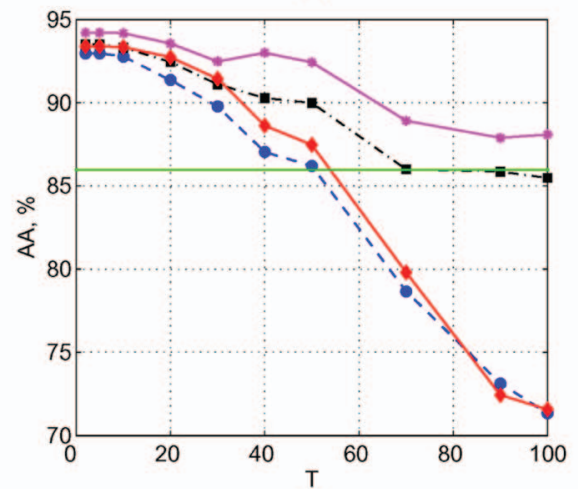

(h)

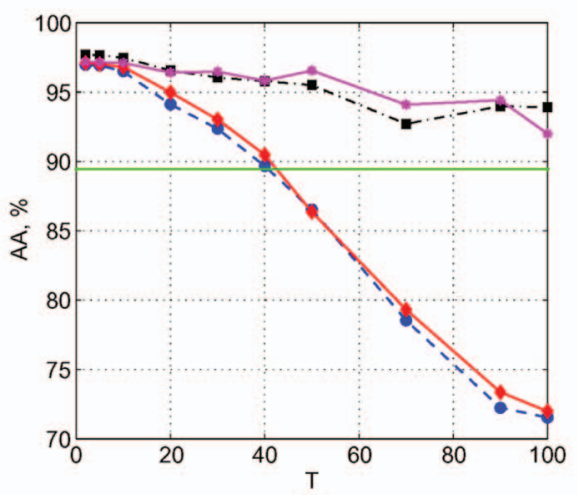

(k)

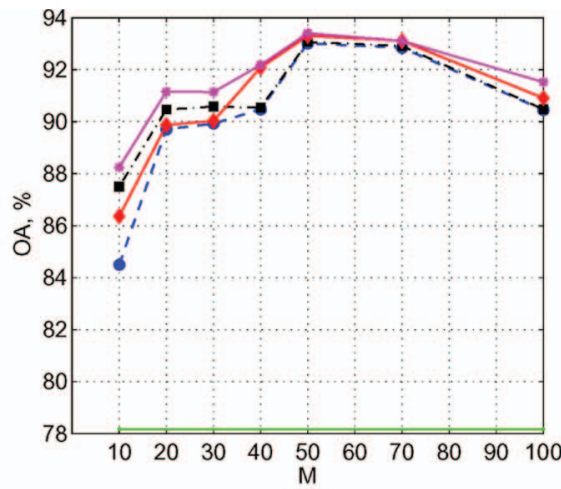

(c)

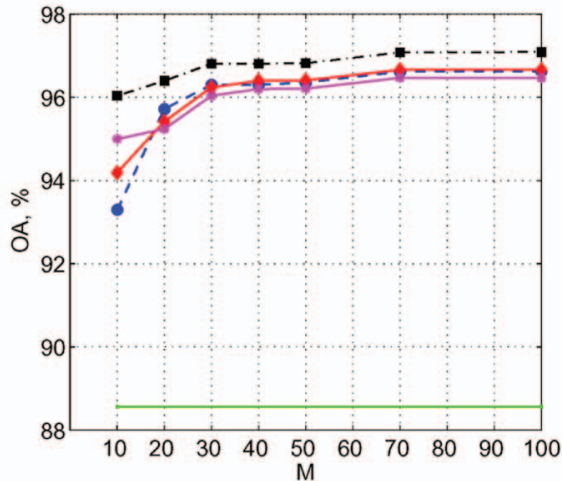

(f)

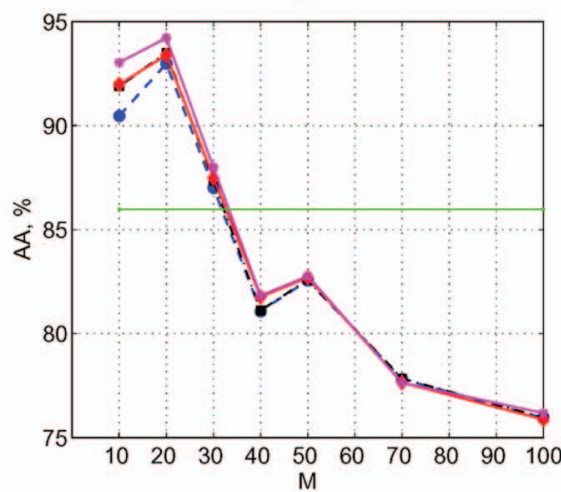

(i)

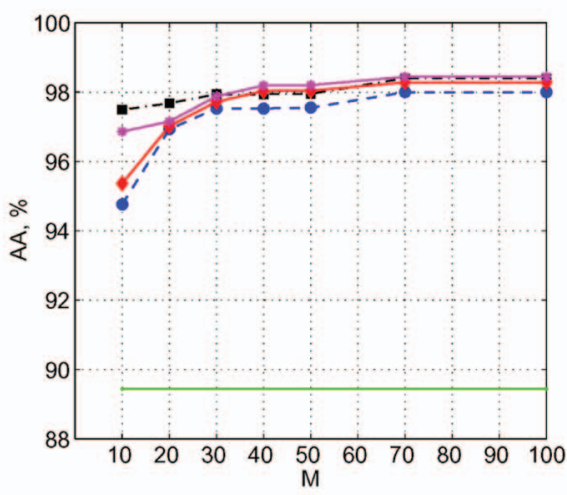

(1)

Fig. 7. Assessment of the robustness of the parameter settings. (a)-(f) Overall and (g)-(l) average classification accuracies as functions of parameters for the marker selection procedure [(a), (d), (g), (j)] $P(M=20, T=2),[(\mathrm{b}),(\mathrm{e}),(\mathrm{h}),(\mathrm{k})] T(M=20, P=20)$, and $[(\mathrm{c}),(\mathrm{f}),(\mathrm{i}),(\mathrm{l})] M(P=20, T=5)$, for the [(a)-(c), (g)-(i)] Indiana and [(d)-(f), (j)-(1)] Hekla images.

norm and the SAM dissimilarity measures were used. Fig. 7 shows the obtained overall [Fig. 7(a) and (d)] and average [Fig. 7(g) and (j)] classification accuracies for both data sets. As can be seen from the figures, for any value of $P$, the accuracies are significantly improved when compared to the pixelwise classification. When the value of $P$ is low (a few marker pixels 
are chosen), the construction of the MSF from the markers gives less accurate classification maps than when it is high. However, the postprocessing majority voting step improves the accuracies significantly. Thus, the complete classification procedure gives the best performances for a low value of $P$. Then, with the increase of $P$, the classification accuracies converge to a constant value, for both dissimilarity measures, and the majority step does not additionally increase the accuracies. Results are coherent for both images. As a conclusion, this parameter has no significant influence on the classification accuracies. When a few marker pixels are chosen (for low values of $P$ ), the construction of an MSF using the SAM distance gives better segmentation results (provides more accurate regions) than a pixelwise classification. Therefore, after the additional processing of the corresponding classification maps by majority voting, the best classification results are achieved.

The choice of the parameter $T$ is investigated, by fixing parameters $M=20$ and $P=2$, varying $T$ from 2 to 100 , and performing classification for both data sets. The overall and average classification accuracies are shown in Fig. 7 (second column). As can be seen, the results are coherent for both dissimilarity measures and both data sets. The highest classification accuracies are achieved for the low values of $T$. These results are coherent with the theoretical analysis given in the previous sections. Since the Indiana and the Hekla images contain large spatial structures (of crop fields and lava formations, respectively), markers for small regions must be selected only in the case if the probability of their correct classification is very high. Therefore, with the increase of $T$, the probability of selecting a marker for a misclassified region increases, and the classification accuracies decrease. The majority voting step becomes more important for higher values of $T$, and for the Hekla image, the accuracies for the complete proposed procedure (including the majority voting step) remain almost unchanged, independently of the parameter $T$.

Furthermore, the choice of the parameter $M$ is investigated, with the other parameters being fixed $(P=20$ and $T=5)$. Fig. 7 (third column) shows the overall and average accuracies of classification when varying the parameter $M$ from 10 to 100. For the Hekla image, the accuracies are slightly lower for $M=10$, when compared to other values of this parameter, for which the accuracies remain almost unchanged. This is due to the fact that the image contains mostly large regions of lava fields. Therefore, for any value of $M$ higher than 20, the classification procedure shows a good performance. For the Indiana image, with the increase of the parameter $M$ up to 50, the overall accuracy slightly increases. However, the average accuracy reaches its maximum for $M=20$ and then significantly decreases. These results confirm the theoretical analysis: The smallest crop field in the image scene has a size of 20 pixels. When a high value of $M$ for identifying significant regions is chosen, small crop fields risk to be assimilated with the neighboring structures. If this happens, majority voting cannot reconstitute these regions, and they disappear from the final classification map.

In conclusion, the experimental analysis of the parameter choice for the marker selection procedure has confirmed that, by using some a priori information for the image, pa- rameters leading to good classification performances can be deducted.

\section{CONCLUSION}

A large number of spectral channels in a hyperspectral image increase the potential of discriminating physical materials and structures in a scene. However, it presents challenges to image analysis because of the huge volume of data that the hyperspectral image usually consists of. Although pixelwise classification techniques have given high classification accuracies when dealing with hyperspectral data, the incorporation of the spatial information into the classifier is needed for further improvement of the classification accuracies.

In this paper, a new spectral-spatial classification scheme for hyperspectral images has been proposed. The method is based on the construction of an MSF, rooted on the markers selected by using pixelwise classification results. Experimental results, presented on the three data sets, have shown that the proposed method improves the classification accuracies, when compared to previously proposed classification schemes, and provides accurate segmentation and classification maps.

Different distances have been investigated for measuring the dissimilarity between pixels when constructing an MSF. It is shown that, in most of the cases, the SAM distance gives the best performances. However, this distance does not discriminate some particular classes from urban areas, such as shadows, and assimilate the pixels belonging to these classes with the neighboring structures.

In conclusion, the proposed classification methodology succeeded in taking advantage of the spatial and the spectral information simultaneously. The method performs well for images representing different scenes: those containing large spatial structures with spectrally confusing classes and those containing small and complex structures. Furthermore, its efficient implementation is possible.

\section{ACKNOWLEDGMENT}

The authors would like to thank P. Gamba and D. Landgrebe for providing the hyperspectral data.

\section{REFERENCES}

[1] R. Jain, R. Kasturi, and B. G. Schunck, Machine Vision. New York: McGraw-Hill, 1995.

[2] C.-I Chang, Hyperspectral Data Exploitation: Theory and Applications. New York: Wiley-Interscience, 2007.

[3] D. A. Landgrebe, Signal Theory Methods in Multispectral Remote Sensing. New York: Wiley, 2003.

[4] P. K. Goel, S. O. Prasher, R. M. Patel, J. A. Landry, R. B. Bonnell, and A. A. Viau, "Classification of hyperspectral data by decision trees and artificial neural networks to identify weed stress and nitrogen status of corn," Comput. Electron. Agric., vol. 39, no. 2, pp. 67-93, 2003.

[5] G. Camps-Valls and L. Bruzzone, "Kernel-based methods for hyperspectral image classification," IEEE Trans. Geosci. Remote Sens., vol. 43, no. 6, pp. 1351-1362, Jun. 2005.

[6] M. Fauvel, "Spectral and spatial methods for the classification of urban remote sensing data," Ph.D. dissertation, Grenoble Inst. Technol., Grenoble, France, 2007.

[7] M. Fauvel, J. Chanussot, J. A. Benediktsson, and J. R. Sveinsson, "Spectral and spatial classification of hyperspectral data using SVMs and morphological profiles," IEEE Trans. Geosci. Remote Sens., vol. 46, no. 11, pp. 3804-3814, Nov. 2008. 
[8] A. Farag, R. Mohamed, and A. El-Baz, "A unified framework for map estimation in remote sensing image segmentation," IEEE Trans. Geosci. Remote Sens., vol. 43, no. 7, pp. 1617-1634, Jul. 2005.

[9] Y. Tarabalka, J. A. Benediktsson, and J. Chanussot, "Classification of hyperspectral data using support vector machines and adaptive neighborhoods," in Proc. 6th EARSeL SIG IS Workshop, Tel Aviv, Israel, 2009.

[10] M. Zhang, L. O. Hall, and D. B. Goldgof, "A generic knowledge-guided image segmentation and labeling system using fuzzy clustering algorithms," IEEE Trans. Syst., Man, Cybern. B, Cybern., vol. 32, no. 5, pp. 571-582, Oct. 2002.

[11] S. Makrogiannis, G. Economou, and S. Fotopoulos, "A region dissimilarity relation that combines feature-space and spatial information for color image segmentation," IEEE Trans. Syst., Man, Cybern. B, Cybern., vol. 35, no. 1, pp. 44-53, Feb. 2005.

[12] R. Gonzalez and R. Woods, Digital Image Processing, 2nd ed. Englewood Cliffs, NJ: Prentice-Hall, 2002.

[13] Y. Tarabalka, J. Chanussot, J. A. Benediktsson, J. Angulo, and M. Fauvel, "Segmentation and classification of hyperspectral data using watershed," in Proc. IGARSS, Boston, MA, 2008, pp. III-652-III-655.

[14] Y. Tarabalka, J. A. Benediktsson, and J. Chanussot, "Spectral-spatial classification of hyperspectral imagery based on partitional clustering techniques," IEEE Trans. Geosci. Remote Sens., vol. 47, no. 8, pp. 29732987, Aug. 2009.

[15] P. Soille, Morphological Image Analysis, 2nd ed. New York: SpringerVerlag, 2003.

[16] A. C. Jalba, M. H. Wilkinson, and J. B. T. M. Roerdink, "Automatic segmentation of diatom images for classification," Microsc. Res. Tech., vol. 65 , no. $1 / 2$, pp. $72-85$, Sep. 2004

[17] G. Noyel, J. Angulo, and D. Jeulin, "Morphological segmentation of hyperspectral images," Image Anal. Stereol., vol. 26, pp. 101-109, 2007.

[18] O. Gómez, J. A. González, and E. F. Morales, "Image segmentation using automatic seeded region growing and instance-based learning," in Proc. 12th Iberoamericann Congr. Pattern Recog., Valparaiso, Chile, Nov. 2007, pp. 192-201.

[19] G. Noyel, "Filtrage, Réduction de Dimension, Classification et Segmentation Morphologique Hyperspectrale," Ph.D. dissertation, Center Math. Morphology, Paris School Mines, Fontainebleau, France, 2008.

[20] L. Kaufman and P. J. Rousseeuw, Finding Groups in Data. An Introduction to Cluster Analysis. Hoboken, NJ: Wiley, 1990.

[21] Y. Tarabalka, J. Chanussot, and J. A. Benediktsson, "Classification based marker selection for watershed transform of hyperspectral images," in Proc. IGARSS, Cape Town, South Africa, 2009.

[22] J. Stawiaski, "Mathematical morphology and graphs: Application to interactive medical image segmentation," Ph.D. dissertation, Paris School Mines, Paris, France, 2008.

[23] O. J. Morris, M. J. Lee, and A. G. Constantinides, "Graph theory for image analysis: An approach based on the shortest spanning tree," Proc. Inst. Elect. Eng.-Commun., Radar Signal Process., vol. 133, no. 2, pp. 146152, Apr. 1986.

[24] J. Shi and J. Malik, "Normalized cuts and image segmentation," IEEE Trans. Pattern Anal. Mach. Intell., vol. 22, no. 8, pp. 888-905, Aug. 2000.

[25] W. Tao, H. Jin, and Y. Zhang, "Color image segmentation based on mean shift and normalized cuts," IEEE Trans. Syst., Man, Cybern. B, Cybern., vol. 37, no. 5, pp. 1382-1389, Oct. 2007.

[26] C. Allène, J. Audibert, M. Couprie, J. Cousty, and R. Keriven, "Some links between min-cuts, optimal spanning forests and watersheds," in Proc. 8th Int. Symp. Math. Morphol., Rio de Janeiro, Brazil, Oct. 2007, pp. 253-264.

[27] C. Couprie, L. Grady, L. Najman, and H. Talbot, "Power watersheds: A new image segmentation framework extending graph cuts, random walker and optimal spanning forest," in Proc. ICCV, Kyoto, Japan, 2009, pp. 731-738.

[28] V. Vapnik, Statistical Learning Theory. New York: Wiley, 1998.

[29] M. Fauvel, J. Chanussot, and J. A. Benediktsson, "Evaluation of kernels for multiclass classification of hyperspectral remote sensing data," in Proc. ICASSP, May 2006, pp. II-813-II-816.

[30] G. Licciardi, F. Pacifici, D. Tuia, S. Prasad, T. West, F. Giacco, J. Inglada, E. Christophe, J. Chanussot, and P. Gamba, "Decision fusion for the classification of hyperspectral data: Outcome of the 2008 GRS-S data fusion contest," IEEE Trans. Geosci. Remote Sens., vol. 47, no. 11, pp. 38573865, Nov. 2009.

[31] T.-F. Wu, C.-J. Lin, and R. C. Weng, "Probability estimates for multi-class classification by pairwise coupling," J. Mach. Learn. Res., vol. 5, pp. 9751005, Dec. 2004.

[32] C. Chang and C. Lin, LIBSVM: A Library for Support Vector Machines, 2001. [Online]. Available: http://www.csie.ntu.edu.tw/ cjlin/ libsvm
[33] H.-T. Lin, C.-J. Lin, and R. C. Weng, "A note on Platt's probabilistic outputs for support vector machines,” Dept. Comput. Sci., Nat. Taiwan Univ., Taipei, Taiwan, 2003.

[34] J. Platt, "Probabilistic outputs for support vector machines and comparison to regularized likelihood methods," in Advances in Large Margin Classifiers, A. Smola, P. Bartlett, B. Schölkopf, and D. Schuurmans, Eds. Cambridge, MA: MIT Press, 2000.

[35] L. Shapiro and G. Stockman, Computer Vision. Englewood Cliffs, NJ: Prentice-Hall, 2002.

[36] F. van der Meer, "The effectiveness of spectral similarity measures for the analysis of hyperspectral imagery," Int. J. Appl. Earth Observation Geoinformation, vol. 8, no. 1, pp. 3-17, 2006.

[37] C.-I Chang, "An information theoretic-based approach to spectral variability, similarity and discriminability for hyperspectral image analysis," IEEE Trans. Inf. Theory, vol. 46, no. 5, pp. 1927-1932, Aug. 2000.

[38] P. Corsini, B. Lazzerini, and F. Marcelloni, "A fuzzy relational clustering algorithm based on a dissimilarity measure extracted from data," IEEE Trans. Syst., Man, Cybern. B, Cybern., vol. 34, no. 1, pp. 775-781, Feb. 2004.

[39] R. C. Prim, "Shortest connection networks and some generalizations," Bell Syst. Tech. J., vol. 36, pp. 1389-1401, 1957.

[40] T. H. Cormen, C. E. Leiserson, R. L. Rivest, and C. Stein, Introduction to Algorithms, 2nd ed. Cambridge, MA: MIT Press, 2001.

[41] A. Widayati, B. Verbist, and A. Meijerink, "Application of combined pixel-based and spatial-based approaches for improved mixed vegetation classification using IKONOS," in Proc. 23rd Asian Conf. Remote Sens., 2002, p. 8.

[42] S. Tadjudin and D. A. Landgrebe, "Covariance estimation with limited training samples," IEEE Trans. Geosci. Remote Sens., vol. 37, no. 4, pp. 2113-2118, Jul. 1999.

[43] G. M. Foody, "Thematic map comparison: Evaluating the statistical significance of differences in classification accuracy," Photogramm. Eng. Remote Sens., vol. 70, no. 5, pp. 627-633, 2004.

[44] J. A. Benediktsson and I. Kanellopoulos, "Classification of multisource and hyperspectral data based on decision fusion," IEEE Trans. Geosci. Remote Sens., vol. 37, no. 3, pp. 1367-1377, May 1999.

[45] A. Plaza, J. A. Benediktsson, J. Boardman, J. Brazile, L. Bruzzone, G. Camps-Valls, J. Chanussot, M. Fauvel, P. Gamba, J. A. Gualtieri, M. Marconcini, J. C. Tilton, and G. Trianni, "Recent advances in techniques for hyperspectral image processing," Remote Sens. Environ., vol. 113, pp. S110-S122, 2009, Supplement 1.

[46] J. A. Benediktsson, J. A. Palmason, and J. R. Sveinsson, "Classification of hyperspectral data from urban areas based on extended morphological profiles," IEEE Trans. Geosci. Remote Sens., vol. 43, no. 3, pp. 480-491, Mar. 2005.

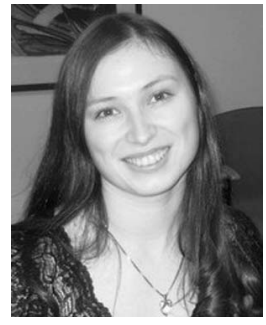

Yuliya Tarabalka (S'08) received the B.S. degree in computer science from Ternopil State Ivan Pul'uj Technical University, Ternopil, Ukraine, in 2005 and the M.Sc. degree in signal and image processing from the Grenoble Institute of Technology (INPG), Grenoble, France, in 2007. She is currently working toward the Ph.D. degree at the University of Iceland, Reykjavik, Iceland, and at INPG.

From July 2007 to January 2008, she was a Researcher with the Norwegian Defence Research Establishment, Norway. Her current research work is funded by the "Hyper-I-Net" Marie Curie Research Training Network. Her research interests are in the areas of image processing, pattern recognition, hyperspectral imaging, and development of efficient algorithms. 


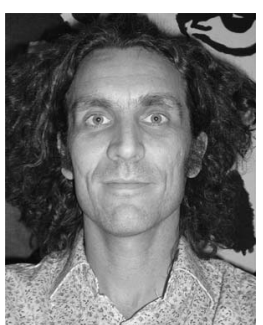

Jocelyn Chanussot (SM'04) received the M.Sc. degree in electrical engineering from the Grenoble Institute of Technology (INPG), Grenoble, France, in 1995 and the Ph.D. degree from the University of Savoie, Annecy, France, in 1998.

In 1999, he was with the Geography Imagery Perception Laboratory, Delegation Generale de l'Armement (French National Defense Department). Since 1999, he has been with INPG, where he was an Assistant Professor from 1999 to 2005, was an Associate Professor from 2005 to 2007, and is currently a Professor of signal and image processing. He is currently conducting his research at the Grenoble Images Speech Signals and Automatics Laboratory (GIPSA Lab), INPG. His research interests include image analysis, multicomponent image processing, nonlinear filtering, and data fusion in remote sensing.

Dr. Chanussot is an Associate Editor for the IEEE TRANSACTIONS ON Geoscience and Remote Sensing. He was the Cochair of the GRS Data Fusion Technical Committee (2005-2008) and a member of the Machine Learning for Signal Processing Technical Committee of the IEEE Signal Processing Society (2006-2008). He was the Founding President of the IEEE GRS French Chapter (2007).

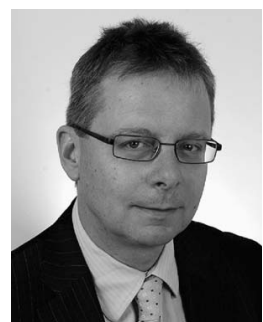

fields.
Jón Atli Benediktsson (F’04) received the Cand.Sci. degree in electrical engineering from the University of Iceland, Reykjavik, Iceland, in 1984 and the M.S.E.E. and Ph.D. degrees from Purdue University, West Lafayette, IN, in 1987 and 1990, respectively.

$\mathrm{He}$ is currently the Pro Rector for Academic Affairs and a Professor of electrical and computer engineering with the University of Iceland. His research interests are in remote sensing, pattern recognition, neural networks, image processing, and signal processing, and he has published extensively in those

Dr. Benediktsson is a member of Societas Scinetiarum Islandica and Tau Beta Pi. He was an Editor of the IEEE TRANS ACTIONS ON GEOSCIENCE AND REMOTE SENSING from 2003 to 2008. He has been on the AdCom of the IEEE Geoscience and Remote Sensing Society (GRSS) since 2000 and is the GRSS Executive Vice President for 2010-2011. He was the recipient of the Stevan J. Kristof Award from Purdue University in 1991 as an outstanding graduate student in remote sensing, the Icelandic Research Council's Outstanding Young Researcher Award in 1997, the IEEE Third Millennium Medal in 2000, the yearly research award from the Engineering Research Institute of the University of Iceland in 2006, and the Outstanding Service Award from the IEEE GRSS in 2007. He was also a corecipient of the University of Iceland's Technology Innovation Award in 2004 